\title{
A Further Characterization on the Sampling Theorem for Wavelet Subspaces ${ }^{\star}$
}

\author{
Xiuzhen $\mathrm{Li}^{1}$ and Deyun Yang${ }^{2}$ \\ ${ }^{1}$ Department of Radiology, Taishan Medical University, Taian 271000, China \\ zhenlixiu@163.com \\ ${ }^{2}$ Department of Information Science and Technology, Taishan University, Taian \\ 271000, China \\ nkuydy@163.com
}

\begin{abstract}
Sampling theory is one of the most powerful results in signal analysis. The objective of sampling is to reconstruct a signal from its samples. Walter extended the Shannon sampling theorem to wavelet subspaces. In this paper we give a further characterization on some shiftinvariant subspaces, especially the closed subspaces on which the sampling theorem holds. For some shift-invariant subspaces with sampling property, the sampling functions are explicitly given.
\end{abstract}

Keywords: Sampling theorem, Wavelet subspace, Wavelet frame, Shift-invariant subspace.

\section{Introduction and Main Results}

Sampling theory is one of the most powerful results in signal analysis. The objective of sampling is to reconstruct a signal from its samples. For example, the classical Shannon theorem says that for each

$$
f \in B_{\frac{1}{2}}=\left\{f \in L^{2}(\mathbf{R}): \operatorname{supp} \widehat{f} \subset\left[-\frac{1}{2}, \frac{1}{2}\right]\right\}
$$

$f(x)=\sum_{k=-\infty}^{\infty} f(k) \frac{\sin \pi(x-k)}{\pi(x-k)}$, where the convergence is both in $L^{2}(\mathbf{R})$ and uniform on $\mathbf{R}$, and the Fourier transform is defined by $\widehat{f}(\omega)=\int_{-\infty}^{\infty} f(x) e^{-2 \pi i x \omega} d x$. If $\psi(x)=\frac{\sin \pi x}{\pi x}$, then $\{\psi(\cdot-k)\}_{k \in \mathbf{Z}}$ is an orthonormal basis for $B_{\frac{1}{2}}$ and $B_{\frac{1}{2}}$ is a shift-invariant subspace in $L^{2}(\mathbf{R})$.

The structure of finitely generated shift-invariant subspaces in $L^{2}(\mathbf{R})$ is studied, e.g., in [1- 6]. There are fruitful results in wavelet theory in the past 20 years (see [7-[12]). In [2], Janssen considered the shifted sampling and corresponding aliasing error by means of Zak transform. Walter [1] extended the Shannon sampling theorem to wavelet subspaces. Zhou and Sun [13] characterized the general shifted wavelet subspaces on which the sampling theorem holds:

\footnotetext{
* This work was supported by the National Natural Science Foundation of China (Grant No. 60572113).
} 
Proposition 1 ([13]). Let $V_{0}$ be a closed subspace in $L^{2}(\mathbf{R})$ and $\{\phi(\cdot-n)\}_{n \in \mathbf{Z}}$ is a frame for $V_{0}$. Then the following two assertions are equivalent:

(i) $\sum_{k} c_{k} \phi(x-k)$ converges pointwise to a continuous function for any $\left\{c_{k}\right\} \in$ $l^{2}$ and there exists a frame $\{S(\cdot-n)\}_{n \in \mathbf{Z}}$ for $V_{0}$ such that

$$
f(x)=\sum_{k} f(k) S(x-k) \text { for any } f \in V_{0},
$$

where the convergence is both in $L^{2}(\mathbf{R})$ and uniform on $\mathbf{R}$.

(ii) $\phi \in C(\mathbf{R}), \sup _{x \in \mathbf{R}} \sum_{k}|\phi(x-k)|^{2}<\infty$ and there exist two positive constants $A, B$ such that

$$
A \chi_{E_{\phi}}(\omega) \leq\left|\sum_{k} \phi(k) e^{-i k \omega}\right| \leq B \chi_{E_{\phi}}(\omega), \text { a.e. },
$$

where $\chi$ is the characteristic function, $E_{\phi}=\left\{\omega \in \mathbf{R}: \sum_{k}|\widehat{\phi}(\omega+2 k \pi)|^{2}>0\right\}$.

Moreover, it implies that for $S$ in $(i), f(k)=\langle f, \widetilde{S}(\cdot-n)\rangle$ for any $f \in V_{0}$, where $\widetilde{S}$ is defined in the following Proposition 3 ,

In this paper we characterize some shift-invariant subspaces, especially the closed subspaces in $L^{2}(\mathbf{R})$ such that the sampling theorem holds.

Notations. Firstly, we discuss functions in $L^{2}(\mathbf{R})$. Therefore $f=g$ means that $f(\omega)=g(\omega)$ for almost everywhere $\omega \in \mathbf{R} . C(\mathbf{R})$ is the space of continuous function. $G_{f}(\omega)=\sum_{k}|\widehat{f}(\omega+k)|^{2}$. It is easy to see that $G_{f}$ is defined only a.e.. $E_{f}=\left\{\omega \in \mathbf{R}: G_{f}(\omega)>0\right\}$ for any $f \in L^{2}(\mathbf{R}) \cdot \chi_{E}$ is the characteristic function of the set $E$. $\widehat{f^{*}}(\omega)=\sum_{n} f(n) e^{-i 2 \pi n \omega}$ for any $f \in L^{2}(\mathbf{R})$ with $\sum_{n}|f(n)|^{2}<\infty . V_{f}^{0}=\left\{g: g(\cdot)=\sum_{n} c_{n} f(\cdot-n)\right.$, where the convergence is in $L^{2}(\mathbf{R})$ and $\left.\left\{c_{n}\right\}_{n \in \mathbf{Z}} \in l^{2}\right\} . V_{f}=\overline{\{f(\cdot-n)\}_{n}}$ which means that any $g \in V_{f}$ can be approximated arbitrarily well in norm by a finite linear combinations of vectors $f(\cdot-n)$. Moreover, $V_{f}$ is called a shift-invariant subspace generated by $f$. Let $\mu(\cdot)$ be the Lebesgue measure on $\mathbf{R}$. For $E, F \subset \mathbf{R}, E \stackrel{a . e}{=} F$ means that $\mu(E \backslash F)=\mu(F \backslash E)=0$.

For $\phi \in L^{2}(\mathbf{R})$, if $\{\phi(\cdot-n)\}_{n}$ is a frame (Riesz basis) for $V_{\phi}$ then $\phi$ is called a frame (Riesz) function.

Definition 1. A closed subspace $V$ in $L^{2}(\mathbf{R})$ is called a sampling space, if there exists a frame $\{\psi(\cdot-k)\}_{k \in \mathbf{Z}}$ for $V$ such that $\sum_{k} c_{k} \psi(x-k)$ converges pointwisely to a continuous function for any $\left\{c_{k}\right\} \in l^{2}$ and

$$
f(x)=\sum_{k} f(k) \psi(x-k) \text { for any } f \in V,
$$

where the convergence is both in $L^{2}(\mathbf{R})$ and uniform on $\mathbf{R}$.

In this case, $\psi$ is called a sampling function on $V$. 
From the definition, we know that if $V$ is a sampling space then for any $f \in V$ there exists a function $g \in C(\mathbf{R})$ such that $f(x)=g(x)$, a.e. $x \in \mathbf{R}$. Therefore, in what follows we assume that all the functions in a sampling space are continuous.

Next we denote the following three sets to characterize the functions with sampling property.

$\mathcal{P}_{1}$ is the subset in $L^{2}(\mathbf{R}) \cap C(\mathbf{R})$ in which each function $\phi$ satisfies that $\sum_{k} c_{k} \phi(x-k)$ converges pointwisely to a continuous function for any $\left\{c_{k}\right\} \in l^{2}$.

$\mathcal{P}_{2}$ is the set of functions in which each function $f$ satisfies the following three conditions:

(i) $f \neq 0, f \in L^{2}(\mathbf{R}) \cap C(\mathbf{R}), \sum_{k}|f(k)|^{2}<\infty$ and $f$ is a bounded function;

(ii) $\mu\left(\left\{\omega: \widehat{f}^{*}(\omega)=0, G_{f}(\omega) \neq 0\right\}\right)=0$;

(iii) There exist two positive constants $A, B$ such that

$$
A \leq \frac{G_{f}(\omega)}{\left|\hat{f}^{*}(\omega)\right|^{2}} \leq B \text {, a.e. } \omega \in E_{f} .
$$

$\mathcal{P}_{3}=\left\{\phi \in \mathcal{P}_{2}: \sup _{x \in \mathbf{R}} \sum_{n}|\phi(x-n)|^{2}<\infty\right\}$.

For any $f \in \mathcal{P}_{2}$, it is easy to see that $\mu\left(E_{f}\right)>0$ and $\widehat{f}^{*}(\omega), G_{f}(\omega)$ are well defined almost everywhere.

Next we list the following propositions to be used in the proof of our results.

Proposition $2\left([3,[5])\right.$. Let $\phi \in L^{2}(\mathbf{R}) . \phi$ is a frame function if and only if there are constants $A, B>0$ such that $A \chi_{E_{\phi}} \leq G_{\phi} \leq B \chi_{E_{\phi}}$, a.e.

Especially, if $\phi$ is a Riesz function, then $E_{\phi}=\mathbf{R}$.

Proposition $3\left([3,13)\right.$. Assume that $f \in L^{2}(\mathbf{R})$ and $f$ is a frame function. Let

$$
\widehat{\widetilde{f}}(\omega)=\left\{\begin{array}{cl}
\frac{\widehat{f}(\omega)}{G_{f}(\omega)}, & \text { if } \omega \in E_{f} \\
0, & \text { if } \omega \notin E_{f}
\end{array}\right.
$$

then $\{\tilde{f}(\cdot-n)\}_{n}$ is a dual frame of $\{f(\cdot-n)\}_{n}$ in $V_{f}$.

Proposition 4 ([13]). Let $\phi \in L^{2}(\mathbf{R})$. Then $\phi \in \mathcal{P}$ if and only if the following holds

(i) $\phi \in C(\mathbf{R})$,

(ii) $\sum_{k \in \mathbf{Z}}|\phi(x-k)|^{2} \leq M$ for some constant $M>0$.

Proposition 5 ([5]). $V_{f}=\left\{g \in L^{2}(\mathbf{R}): \widehat{g}=\tau \widehat{f}\right.$, where $\tau$ is a function with period 1 and $\left.\tau \widehat{f} \in L^{2}(\mathbf{R})\right\}$.

Proposition 6 ([5]). Let $g \in V_{f}$. Then $V_{g}=V_{f}$ if and only if supp $\widehat{f} \stackrel{\text { a.e }}{=}$ supp $\widehat{g}$.

Now we give the following theorem to check whether $V_{f}$ is the maximum shiftinvariant subspace in $L^{2}(\mathbf{R})$. 
Theorem 7. Let $f \in L^{2}(\mathbf{R})$. There exists $g \in L^{2}(\mathbf{R})$ such that $f \in V_{g}$ and $g \notin V_{f}$ if and only if there exists $E \subseteq[0,1]$ with $\mu(E)>0$ such that $\widehat{f}(\omega)=0$ for any $\omega \in \bigcup_{k \in \mathbf{Z}}(k+E)$.

For a shift-invariant subspace $V_{f}$ with sampling property, how can we find its sampling functions? For this, we give the following two theorems. Firstly, for any $f \in L^{2}(\mathbf{R})$ if $\widehat{f^{*}}$ is well defined, then we define

$$
\widehat{f}_{p}(\omega)=\left\{\begin{array}{ccc}
\frac{\widehat{f}(\omega)}{\hat{f}^{*}(\omega)}, & \text { if } & \widehat{f}^{*}(\omega) \neq 0, \\
0, & \text { if } & \widehat{f}^{*}(\omega)=0 .
\end{array}\right.
$$

Theorem 8. If $f \in \mathcal{P}_{2}$, then $\left\{f_{p}(\cdot-n)\right\}_{n}$ is a frame for $V_{f}$.

Theorem 9. (i) Let $\hat{f}^{*}(\omega) \neq 0$, a.e. $\omega \in \mathbf{R}$. If $S \in L^{2}(\mathbf{R})$ such that

$$
f(x)=\sum_{k} f(k) S(x-k),
$$

where the convergence is in $L^{2}(\mathbf{R})$, then $S=f_{p}$.

(ii) For any $f \in \mathcal{P}_{3}$, let

$$
\widehat{S}(\omega)=\left\{\begin{array}{l}
\frac{\widehat{f}(\omega)}{\hat{f}^{*}(\omega)}, \text { if } \widehat{f}^{*}(\omega) \neq 0, \\
R(\omega), \text { if } \widehat{f}^{*}(\omega)=0,
\end{array}\right.
$$

where $R \in L^{2}(\mathbf{R})$. Then for any $g \in V_{f}^{0}, g(x)=\sum_{n} g(n) S(x-n)$.

Finally, we give the following equivalent characterization on shift-invariant subspaces with sampling property.

Theorem 10. Assume that $V$ is a shift-invariant subspace. Then the following assertions are equivalent:

(i) $V$ is a sampling space.

(ii) For any $\phi \in C(\mathbf{R})$ such that $\{\phi(\cdot-n)\}_{n}$ is a frame for $V$, we have

$$
\sup _{x} \sum_{n}|\phi(x-n)|^{2}<\infty,
$$

and there exist positive constants $A, B$ such that

$$
A \chi_{E_{\phi}}(\omega) \leq\left|\widehat{\phi}^{*}(\omega)\right| \leq B \chi_{E_{\phi}}(\omega) \text {, a.e. }
$$

(iii) There exists $\phi \in C(\mathbf{R})$ such that $\{\phi(\cdot-n)\}_{n}$ is a frame for $V$ and (2), (3) hold.

(iv) There exists $\phi \in C(\mathbf{R})$ such that $\{\phi(\cdot-n)\}_{n}$ is a frame for $V$, (2) holds and

$$
A\|g\|^{2} \leq \sum_{n}|g(n)|^{2} \leq B\|g\|^{2}, \forall g \in V .
$$

for some positive constants $A, B$.

(v) There exists $\phi \in C(\mathbf{R})$ such that $\{\phi(\cdot-n)\}_{n}$ is a frame for $V$, (2) holds and $\left\{\sum_{l} \overline{\phi(k-l)} \widetilde{\phi}(k-\cdot)\right\}_{k}$ is a frame for $V$. 


\section{Proof of Main Results}

Proof of Theorem [7. [necessary] Assume that $g \in L^{2}(\mathbf{R}), f \in V_{g}$ and $g \notin V_{f}$. By Proposition [5 and Proposition [6, $\widehat{f}=\tau \widehat{g}$ where $\tau$ is a a function with period 1 and $\mu(\operatorname{supp} \widehat{g} \backslash$ supp $\widehat{f})>0$. Then there exists $E^{*} \in \mathbf{R}$ with $\mu\left(E^{*}\right)>0$ such that $\widehat{g}(\omega) \neq 0, \widehat{f}(\omega)=0$ for any $\omega \in E^{*}$. Thus $\tau(\omega)=0$ for any $\omega \in E^{*}$. Let

$$
T\left(E^{*}\right)=\left\{\omega: \omega \in[0,1] \text {, there exists } k \in \mathbf{Z} \text { such that } \omega+k \in E^{*}\right\} .
$$

Then $\tau(\omega)=0$ for any $\omega \in T\left(E^{*}\right)$. Since $E^{*} \subseteq \bigcup_{k \in \mathbf{Z}}\left(k+T\left(E^{*}\right)\right)$ and $\mu\left(E^{*}\right)>0$, we have $\mu\left(T\left(E^{*}\right)\right)>0$. Therefore, if we take $E=T\left(E^{*}\right)$, then $E$ satisfies the conditions in the theorem.

[sufficiency] Assume that $f \in L^{2}(\mathbf{R})$ and there exists $E \subseteq[0,1]$ with $\mu(E)>0$ such that $\widehat{f}(\omega)=0$ for any $\omega \in \bigcup_{k \in \mathbf{Z}} k+E$. Let

$$
\widehat{g}(\omega)=\left\{\begin{array}{c}
\widehat{f}(\omega), \text { if } \omega \notin E, \\
1, \quad \text { if } \omega \in E .
\end{array}\right.
$$

Then $\widehat{f}(\omega)=\tau(\omega) \widehat{g}(\omega)$ for any $\omega \in \mathbf{R}$, where

$$
\tau(\omega)=\left\{\begin{array}{l}
1, \text { if } \omega \notin \bigcup_{k \in \mathbf{Z}}(k+E), \\
0, \text { if } \omega \in \bigcup_{k \in \mathbf{Z}}(k+E)
\end{array}\right.
$$

is a function with period 1 and $\mu(\operatorname{supp} \widehat{g} \backslash \operatorname{supp} \widehat{f})>0$. Thus we have $f \in V_{g}$ and $g \notin V_{f}$.

This completes the proof of Theorem 7

Proof of Theorem [8, By (1) and $f \in \mathcal{P}_{2}$, we have $\widehat{f}_{p}(\omega)=\tau(\omega) \widehat{f}(\omega)$, where

$$
\tau(\omega)=\left\{\begin{array}{cl}
\frac{1}{\hat{f}^{*}(\omega)}, & \text { if } \omega \in E_{f} \\
0, & \text { if } \omega \notin E_{f}
\end{array}\right.
$$

Then

$$
G_{f_{p}}(\omega)=\left\{\begin{array}{cl}
\frac{G_{f}(\omega)}{\left|\hat{f}^{*}(\omega)\right|^{2}}, & \text { if } \omega \in E_{f} \\
0, & \text { if } \omega \notin E_{f} .
\end{array}\right.
$$

Since $f \in \mathcal{P}_{2}$, there exist positive constants $A, B$ such that $A \chi_{E_{f}} \leq G_{f_{p}} \leq B \chi_{E_{f}}$. However, $\tau$ is a function with period 1. By Proposition 5, $f_{p} \in V_{f}$. It is from supp $f=\operatorname{supp}_{p}$ and Proposition 6 that $V_{f}=V_{f_{p}}$. Finally, by Proposition 2, $f_{p}$ is a frame for $V_{f}$.

This completes the proof of Theorem 8 .

Proof of Theorem 9. [(i)] Since $f(x)=\sum_{k} f(k) S(x-k)$ and $\widehat{f}^{*}(\omega) \neq 0$, we have $\widehat{f}(\omega)=\widehat{f}^{*}(\omega) \widehat{S}(\omega), \widehat{S}(\omega)=\frac{\widehat{f}(\omega)}{\hat{f}^{*}(\omega)}$. Thus $S=f_{p}$.

$[(i i)]$ For any $g \in V_{f}^{0}$, there exist $\left\{c_{k}\right\} \in l^{2}$ such that $g(\cdot)=\sum_{k} c_{k} f(\cdot-k)$, where the convergence is pointwise and $g \in C(\mathbf{R})$. Let $C(\omega)=\sum_{k} c_{k} e^{-i 2 \pi k \omega}$, 
$C^{*}(\omega)=C(\omega) \chi_{E_{f}}(\omega)$. Then there exists $\left\{c_{k}^{*}\right\} \in l^{2}$ such that $C^{*}(\omega)=$ $\sum_{k} c_{k}^{*} e^{-i 2 \pi k \omega}$. It follows from

$$
\widehat{g}(\omega)=C(\omega) \widehat{f}(\omega)=C^{*}(\omega) \widehat{f}(\omega)
$$

and Proposition 4 that $g(x)=\sum_{k} c_{k}^{*} f(x-k)$ converges pointwisely in $\mathbf{R}$. By Theorem 8, $f_{p}$ is a frame for $V_{f}$. Then using Proposition $3, \widetilde{f}_{p}$ is a dual frame of $\{f(\cdot-n)\}_{n \in \mathbf{Z}}$ in $V_{f}$. Thus

$$
\begin{aligned}
\left(g, \widetilde{f}_{p}(\cdot-n)\right) & =\int_{E_{f}} C^{*}(\omega) \frac{\widehat{f}^{*}(\omega)}{G_{f}(\omega)}|\widehat{f}(\omega)|^{2} e^{i 2 \pi n \omega} d \omega=\int_{-1 / 2}^{1 / 2} C^{*}(\omega) \widehat{f}^{*}(\omega) e^{i 2 \pi n \omega} d \omega \\
& =\sum_{k} c_{k}^{*} f(n-k)=g(n) .
\end{aligned}
$$

Thus we get $g(x)=\sum_{n} g(n) f_{p}(x-n)$. Let $\widehat{h}(\omega)=R(\omega) \chi_{\left\{\omega: \widehat{f}^{*}(\omega)=0\right\}}$. Then

$$
\widehat{S}(\omega)=\widehat{f}_{p}(\omega)+\widehat{h}(\omega), S=f_{p}+h .
$$

Since $g(n)=\sum_{k} c_{k}^{*} f(n-k)$ for $n \in \mathbf{Z}$, we have $\widehat{g^{*}}(\omega)=\sum_{k} c_{k}^{*} e^{-i 2 \pi k \omega} \widehat{f^{*}}(\omega)$. Then

$$
\widehat{g^{*}}(\omega) \widehat{h}(\omega)=0, \sum_{n} g(n) h(x-n)=0 .
$$

Therefore

$$
g(x)=\sum_{n} g(n)\left(f_{p}(x-n)+h(x-n)\right)=\sum_{n} g(n) S(x-n) .
$$

This completes the proof of Theorem 9.

For the proof of Theorem 10, we give the following Lemma.

Lemma 1. Assume that $\phi \in \mathcal{P}_{1}$ is a frame function. If $f \in V_{\phi}$ satifies $\widehat{f}(\omega)=$ $b(\omega) \widehat{\phi}(\omega)$, where $b(\omega)$ is a function with period 1 and bounded on $E_{\phi}$, then $f \in \mathcal{P}_{1}$. Specially, for any frame function $\psi \in V_{\phi}$, we have $\psi \in \mathcal{P}_{1}$.

Proof. Assume that $f \in V_{\phi}$ satisfies $\widehat{f}(\omega)=b(\omega) \widehat{\phi}(\omega)$, where $b(\omega)$ is a function with period 1 and bounded on $E_{\phi}$. Let $B(\omega)=b(\omega) \chi_{E_{\phi}}(\omega)$. Since $B(\omega)$ is bounded on $\left[-\frac{1}{2}, \frac{1}{2}\right]$, there exists $\left\{B_{n}\right\} \in l^{2}$ such that $B(\omega)=\sum_{n} B_{n} e^{-i 2 \pi n \omega}$. Since $\phi \in \mathcal{P}_{1}$ and

$$
\widehat{f}(\omega)=b(\omega) \widehat{\phi}(\omega)=B(\omega) \widehat{\phi}(\omega),
$$

we have $f \in C(\mathbf{R})$ and $f(x)=\sum_{n} B_{n} \phi(x-n)$, where the convergence is both in $L^{2}(\mathbf{R})$ and pointwisely.

Now using $\phi \in \mathcal{P}_{1}$ and Proposition 4, we have $\sup _{x \in \mathbf{R}} \sum_{n}|\phi(x-n)|^{2}<\infty$. Hence

$$
\sup _{x} \sum_{k}|f(x-k)|^{2}=\sup _{x} \sum_{k}\left|\sum_{n} B_{n} \phi(x-k-n)\right|^{2}
$$




$$
\begin{aligned}
& =\sup _{x} \int_{-1 / 2}^{1 / 2}|B(\omega)|^{2}\left|\sum_{k} \phi(x-k) e^{i 2 \pi k \omega}\right|^{2} d \omega \\
& \leq \sup _{x}\|B(\omega)\|_{\infty}^{2} \sum_{k}|\phi(x-k)|^{2}<\infty .
\end{aligned}
$$

Then by $f \in C(\mathbf{R})$, (5) and Proposition 4, we get $f \in \mathcal{P}_{1}$.

For any frame function $\psi \in V_{\phi}$, there exists a function $\tau$ with period 1 such that $\widehat{\psi}(\omega)=\tau(\omega) \widehat{\phi}(\omega)$, then $G_{\psi}=|\tau(\omega)|^{2} G_{\phi}(\omega)$. By Proposition 2 is bounded on $E_{\phi}$. Thus $\psi \in \mathcal{P}_{1}$.

This completes the proof of Lemma 1

Proof of Theorem of 10. $(i) \Rightarrow(i i)$ Given any continuous function $\phi$ such that $\{\phi(\cdot-n)\}_{n}$ is a frame for sampling space $V$. Let $\psi$ be a sampling function for $V$. By Proposition $4 \sup _{x \in \mathbf{R}} \sum_{n}|\psi(x-n)|^{2}<\infty$.

Let $\widehat{\phi}(\omega)=b(\omega) \widehat{\psi}(\omega)$, where $b(\omega)=\sum_{k} b_{k} e^{-i 2 \pi k \omega}$ for some $\left\{c_{k}\right\} \in l^{2}$. Then $G_{\phi}(\omega)=|b(\omega)|^{2} G_{\psi}(\omega)$. By Proposition 2, $b(\omega)$ is bounded on $E_{\psi}$. By Lemma 1 $\phi \in \mathcal{P}_{1}$. Then by Proposition [1] we get (ii).

$($ ii $) \Rightarrow($ iii $)$ It is trivial.

$($ iii $) \Rightarrow(i)$ By Proposition 1, there exists a frame $\{\psi(\cdot-n)\}_{n}$ for $V$ such that $f(x)=\sum_{n} f(n) \psi(x-n)$ for any $f \in V$. By Lemma 1 and Proposition $4, \psi \in \mathcal{P}_{1}$. Thus we get $(i)$.

$(i v) \Rightarrow(i)$ For any $n \in \mathbf{Z}, T g=g(n)$ is a bounded linear functional on $V$. Then there exists $S_{n} \in V$ such that

$$
g(n)=\left\langle g, S_{n}\right\rangle \text { for any } n \in \mathbf{Z}, g \in V .
$$

Let $S:=S_{0}$ and $g_{1}(x)=g(x+1)$, for any $g \in V, x \in \mathbf{R}$. Then

$$
\left\langle g_{1}, S\right\rangle=g_{1}(0)=g(1)=\left\langle g, S_{1}\right\rangle .
$$

Thus for any $g \in V$, we have

$$
\begin{aligned}
\int g(x) \overline{S(x-1)} d x & =\int g(x+1) \overline{S(x)} d x=\int g_{1}(x) \overline{S(x)} d x=g_{1}(0)=g(1) \\
& =\left\langle g, S_{1}\right\rangle=\int g(x) \overline{S_{1}(x)} d x .
\end{aligned}
$$

Thus $S_{1}(x)=S(x-1)$. Similarly, we have $S_{n}(x)=S(x-n)$ for any $n \in \mathbf{Z}, x \in \mathbf{R}$. Therefore

$$
g(n)=\langle g, S(\cdot-n)\rangle \text { for any } g \in V .
$$

Now by (4), $S$ is a frame for $V$. Let $\widetilde{S}$ be defined as Proposition 3 . Then by Lemma 1 and Proposition $4, \widetilde{S}$ is the sampling function for $V$. Therefore we get $(i)$.

$(i) \Longleftrightarrow(i v)$ Note that in Proposition 1, $f(n)=\langle f, \widetilde{S}(\cdot-n)\rangle$ for any $f \in V_{0}$, where $\{\widetilde{S}(\cdot-n)\}_{n}$ is a frame for $V_{0}$. Thus it is just from Proposition 1 . 
$(i v) \Longleftrightarrow(v)$ Assume that there exists a continuous function $\phi$ which is a frame function for $V$ such that $\sup _{x} \sum_{n}|\phi(x-n)|^{2}<\infty$. Then by Lemma 1 we have $\widetilde{\phi} \in \mathcal{P}_{1}$. Therefore $q_{x}(\cdot):=\sum_{l} \overline{\phi(x-l)} \widetilde{\phi}(\cdot-l)$ convergences both in $L^{2}(\mathbf{R})$ and uniformly on $\mathbf{R}$.

Now for any $g \in V$,

$$
\left\langle g, q_{x}\right\rangle=\sum_{k}\langle g, \widetilde{\phi}(\cdot-k)\rangle \phi(x-k) \text { for any } x \in \mathbf{R} .
$$

The last series converges both in $L^{2}(\mathbf{R})$ to $g$ and uniformly to $\left\langle g, q_{x}\right\rangle$. Thus

$$
g(x)=\left\langle g, q_{x}\right\rangle \text { for any } x \in \mathbf{R} .
$$

If $(i v)$ holds, then there exist two positive constants $A, B$ such that

$$
A\|g\|^{2} \leq \sum_{k}\left|\left\langle g, q_{k}\right\rangle\right|^{2} \leq B\|g\|^{2} \text { for any } g \in V .
$$

Thus $\left\{q_{k}(\cdot)\right\}_{k}=\left\{\sum_{l} \overline{\phi(k-l)} \widetilde{\phi}(k-\cdot)\right\}_{k}$ is a frame for $V$. We get $(v)$.

If $(v)$ holds, then $\left\{q_{k}(\cdot)\right\}_{k}$ is a frame for $V$. By (6) and (77), we get $(i v)$.

This completes the proof of Theorem 10 .

\section{References}

1. Walter,G.: A sampling theorem for wavelet subspaces. IEEE Trans. Inform. Theory 38 (1992) 881-884

2. Janssen, A.J.E.M.: The Zak transform and sampling theorems for wavelet subspaces. J. Fourier Anal. Appl. 2 (1993) 315-327

3. Boor, C., Devore, R., Ron, A.: The structure of finitely generated shift-invariant subspaces in $L^{2}(\mathbf{R})$. J. Func. Anal. 119 (1994) 37-78

4. Boor, C., Devore, R., Ron, A.: Approximation from shift-invariant subspaces of $L^{2}\left(\mathbf{R}^{d}\right)$. Trans. Amer. Math, Soc. 341 (1994) 787-806

5. Benedetto, J.J., Walnut, D.F.: Gabor frames for $L^{2}$ and related spaces. In: Benedetto, J.J., Frazier, M. W. (eds.): Wavelets: Mathematics and Applications. CRC Press, Boca Raton (1993) 1-36

6. Ron, A., Shen, Z.W.: Frames and stable bases for shift-invariant subspaces of $L^{2}\left(\mathbf{R}^{d}\right)$. Can. J. Math. Vol. 5 (1995) 1051-1094

7. Daubechies, I.: Ten lectures on wavelets, Philadalphia, SIAM (1992)

8. Christensen, O: An Introduction to Frames and Riesz Bases. Birkhäuser, Boston (2003)

9. Chui, C., Shi, X.: Orthonormal wavelets and tight frames with arbitrary real dilations, Appl. Comp. Harmonic Anal. 9 (2000) 243-264

10. Yang, D., Zhou, X.: Irregular wavelet frames on $L^{2}\left(\mathbf{R}^{\mathbf{n}}\right)$, Science in China Ser. A. Math. 48 (2005) 277-287

11. Yang, D., Zhou, X.: Wavelet frames with irregular matrix dilations and their stability, J. Math. Anal. Appl. 295 (2004) 97-106

12. Yang, D., Zhou, X.: Frame wavelets with matrix dilations in $L^{2}\left(\mathbf{R}^{\mathbf{n}}\right)$, Appl. Math. Letters 17 (2004) 631-639

13. Zhou, X.W., Sun, W.C: On the sampling theorem for wavelet subspace. J. Fourier Anal. Appl. 1 (1999) 347-354 